\title{
Faulty Estimates of Patients' Body-Weight Affect dose of Intravenous Alteplase without any Impact on Outcome
}

Arvind Sharma ${ }^{1,2}$, P. Paliwal ${ }^{3}$, K. Kumar RN ${ }^{4}$, S. Vrushali B $B^{4},{ }^{2}$

'Zydus Hospital- Ahmedabad, Neurology, Ahmedabad, India

${ }^{2} \mathrm{~B}$ J Medical College - Civil Hospital, Neurology, Ahmedabad, India

${ }^{3}$ National University Health System, Division of Neurology, Singapore, Singapore

${ }^{4}$ Yashoda Hospital- Hyderabad, Neurology, Hyderabad, India

${ }^{5}$ Yong Loo Lin School of Medicine, National University of Singapore, Singapore, Singapore

\section{Background and Aims:}

Intravenously administered tissue plasminogen activator(IV-tPA) remains the main treatment for acute ischemic stroke(AIS). Since early initiation of IV-tPA results in better functional outcome, treatment is initiated, based on the estimated or last-known bodyweight of the patient. This approach may result in underdosing or overdosing of tPA and affect the outcome. In this multicenter retrospective study, we evaluated the extent of error in our AIS cohort and its impact on functional outcome and symptomatic intracranial hemorrhage( $\mathrm{SICH})$.

\section{Method:}

Consecutive AIS patients thrombolysed on the basis of estimated body-weight at 3 tertiary centers between January-to-December 2016 were included. Collected data included information about demographics, cardiovascular risk factors, stroke subtype and National Institute of Health Stroke Scale (NIHSS) scores. Estimated and measured body-weights were recorded. Functional outcome was assessed at 3-months by modified Rankin scale (mRS), the score of 0-1 defined good outcome.

Table 1: Baseline Characteristics of the study population $(n=150)$

\begin{tabular}{|r|r|}
\hline \multicolumn{1}{|c|}{ Variable } & \\
\hline Median Age (IQR) & $64(55-75)$ \\
\hline Male gender- n (\%) & $101(67)$ \\
\hline Race & \\
Chinese- n (\%) & $57(38)$ \\
Indian/Malay/Others- n (\%) & $93(62)$ \\
\hline Diabetes mellitus- n (\%) & $55(36.7)$ \\
\hline Hypertension- n (\%) & $100(66.7)$ \\
\hline Hyperlipidemia- n (\%) & $77(51.3)$ \\
\hline Atrial fibrillation- n (\%) & $24(16)$ \\
\hline Ischemic heart disease- n (\%) & $33(22)$ \\
\hline Smoker- n (\%) & $43(28.6)$ \\
\hline Previous stroke- n (\%) & $17(11.3)$ \\
\hline Median NIHSS on arrival (IQR) & $9(6-17)$ \\
\hline TOAST Classification- n (\%) & $35(23.3)$ \\
Large Artery Disease & $46(30.7)$ \\
Cardio embolism & $42(28)$ \\
Lacunar & $0(0)$ \\
Other determined causes & $27(18)$ \\
\hline Undetermined Etiology & $74(49.3)$ \\
\hline mRS (0-1) at 3 months- n (\%) & $92(61.3)$ \\
\hline mRS (0-2) at 3 months- n (\%) & $10(6.6)$ \\
\hline Symptomatic ICH- n (\%) & $10(6.7)$ \\
\hline Death within 3 months- n (\%)
\end{tabular}
Abbreviations: ICH- intracranial hemorrhage; IQR- interquartile range; mRS- modified Rankin scale; NIHSS- National Institute of Health
Stroke Scale; TOAST- Trial of ORG 10172 in acute stroke treatment

Table 2: Body-Weight estimation in the study population $(n=150)$

\begin{tabular}{|r|r|}
\hline Variable & \\
\hline Median difference between estimated and measured & $3.0(1.5-6.0)$ \\
body-weight in Kg (IQR) & \\
Number of cases with $>5 \mathrm{~kg}$ difference $(\%)$ & $55(36.7)$ \\
Number of cases with $>10 \mathrm{~kg}$ difference $(\%)$ & $23(15.3)$ \\
Number of cases with $>20 \mathrm{~kg}$ difference $(\%)$ & $8(5.3)$ \\
\hline
\end{tabular}

Table 3: Independent predictors for good functional outcome (mRS 0-1) at 3-months

\begin{tabular}{|r|r|r|}
\hline & Odds Ratio (95\% confidence interval) & p-value \\
\hline NIHSS & $1.288(1.157-1.435)$ & 0.000 \\
\hline TOAST & $5.878(1.929-17.910)$ & 0.002 \\
\hline $\begin{array}{r}\text { Difference of }>10 \% \\
\text { between estimated and } \\
\text { measured body-weight }\end{array}$ & $2.436(0.729-8.145)$ & 0.148 \\
& & \\
\hline
\end{tabular}

Table 4: Independent predictors for good outcome at 3-months (mRS 0-1) (after excluding patients with underestimated body-weight)

\begin{tabular}{|r|r|r|}
\hline & Odds Ratio (95\% confidence interval) & P Value \\
\hline NIHSS & $1.306(1.166-1.464)$ & 0.000 \\
\hline TOAST & & 0.007 \\
\hline Large Artery Disease & $4.983(1.154-21.510)$ & 0.031 \\
\hline Difference in weight $>10 \%$ & $1.438(0.304-6.791)$ & 0.647 \\
\hline
\end{tabular}

\section{Results:}

The study included 150 patients. Median age was 64-years (IQR 55-75) with male preponderance(67\%) and median NIHSS score of 9-points (IQR 6-17). Cardioembolism was the commonest stroke subtype(30.7\%). Median difference between actual and estimated body-weight was 3-kg (IQR 1.5-6). Difference was more than $10 \%$ in $35(23.3 \%)$ patients. Good functional outcome was achieved by $74(49.3 \%)$ patients and $10(6.8 \%)$ developed SICH. NIHSS (OR $1.288 ; 95 \% \mathrm{Cl}$ $1.157-1.435, \mathrm{p}<0.001$ ) and large artery atherosclerosis (OR 5.878; $95 \% \mathrm{Cl} 1.929$ $17.910, p=0.002$ ) were independent predictors of unfavorable functional outcome

Table 5. Differences in the characteristics of patients with good (mRS 0-1) and unfavorable (mRS 2-6) functional outcome at 3-months ( $n=150$ ).

\begin{tabular}{|c|c|c|c|}
\hline Variable & $\begin{array}{c}\text { Good outcome } \\
(n=74)\end{array}$ & $\begin{array}{c}\begin{array}{c}\text { Unfavorable outcome } \\
(n=76)\end{array} \\
\end{array}$ & $\begin{array}{c}\text { p- } \\
\text { value }\end{array}$ \\
\hline Median Age in years (IQR) & $61(54-67)$ & $69(56-81)$ & 0.006 \\
\hline Male Gender & $56(75.7)$ & $45(59.2)$ & 0.032 \\
\hline Race- n (\%) & & & 0.006 \\
\hline Chinese & $20(27)$ & $37(48.7)$ & \\
\hline Non-Chinese & $54(73)$ & $39(51.3)$ & \\
\hline Hypertension- $\mathrm{n}(\%)$ & $50(67.6)$ & $50(65.8)$ & 0.817 \\
\hline Diabetes mellitus- $\mathrm{n}(\%)$ & $25(33.8)$ & $30(39.5)$ & 0.470 \\
\hline Dyslipidemia- $\mathrm{n}(\%)$ & $34(45.9)$ & $43(56.6)$ & 0.193 \\
\hline Ischemic heart disease- $\mathrm{n}(\%)$ & $18(24.3)$ & $15(19.7)$ & 0.498 \\
\hline Atrial Fibrillation- $\mathrm{n}(\%)$ & $7(9.4)$ & $17(22.4)$ & 0.031 \\
\hline Smoking- $\mathrm{n}(\%)$ & $23(31.1)$ & $20(26.3)$ & 0.519 \\
\hline Previous stroke- $\mathrm{n}(\%)$ & $10(13.5)$ & $7(9.2)$ & 0.406 \\
\hline Median NIHSS on arrival (IQR) & $6(4-9)$ & $16(10-21)$ & $<0.001$ \\
\hline Stroke subtype- $\mathrm{n}(\%)$ & & & 0.001 \\
\hline LAA & $10(13.5)$ & $25(32.8)$ & \\
\hline Cardioembolism & $17(23)$ & $29(38.2)$ & \\
\hline Lacunar Stroke & $37(50)$ & $5(6.6)$ & \\
\hline Other determined cause & $0(0)$ & $0(0)$ & \\
\hline Undetermined etiology & $10(13.5)$ & $17(22.4)$ & \\
\hline $\begin{array}{r}\text { Difference }(>10 \%) \text { in actual and } \\
\text { estimated weight }\end{array}$ & $10(13.5)$ & $25(32.9)$ & 0.005 \\
\hline
\end{tabular}

Table 6: Differences in the characteristics of patients with good (mRS score $0-1)$ and unfavorable (mRS score 2-6) functional outcome at 3 months (after excluding patients with under estimation of body-weight)

\begin{tabular}{|c|c|c|c|}
\hline Variable & $\begin{array}{r}\text { Good outcome 3- } \\
\text { months }(n=70)\end{array}$ & $\begin{array}{r}\text { Unfavorable } \\
\text { outcome at 3- } \\
\text { months }(n=68)\end{array}$ & p-value \\
\hline Median Age in years (IQR) & $61(54-66)$ & $69(56-81)$ & 0.006 \\
\hline Male Gender- $\mathrm{n}(\%)$ & $52(75.7 \%)$ & $43(59.2 \%)$ & 0.161 \\
\hline Race- $\mathrm{n}(\%)$ & & & 0.006 \\
\hline Chinese & $19(26 \%)$ & $34(50 \%)$ & \\
\hline Non-Chinese & $51(74 \%)$ & $34(50 \%)$ & \\
\hline Hypertension- $\mathrm{n}(\%)$ & $47(67.1 \%)$ & $44(64.7 \%)$ & 0.763 \\
\hline Diabetes mellitus- $\mathrm{n}(\%)$ & $23(32.9 \%)$ & $29(42.7 \%)$ & 0.509 \\
\hline Dyslipidemia- n (\%) & $32(45.7 \%)$ & $38(55.9 \%)$ & 0.193 \\
\hline Ischemic heart disease- $\mathrm{n}(\%)$ & $17(24.3 \%)$ & $12(17.6 \%)$ & 0.339 \\
\hline Atrial Fibrillation- $\mathrm{n}(\%)$ & $6(8.6 \%)$ & $16(23.5 \%)$ & 0.016 \\
\hline Smoking- $\mathrm{n}(\%)$ & $22(31.4 \%)$ & $20(29.4 \%)$ & 0.797 \\
\hline Previous Stroke- $\mathrm{n}(\%)$ & $10(14.3 \%)$ & $6(8.8 \%)$ & 0.316 \\
\hline Median NIHSS on arrival (IQR) & $7(4-9)$ & $17(10-21)$ & 0.000 \\
\hline Stroke subtype- $\mathrm{n}(\%)$ & & & 0.000 \\
\hline Large Artery Atherosclerosis & $9(12.9 \%)$ & $22(32.4 \%)$ & \\
\hline Cardioembolism & $16(22.9 \%)$ & $28(41.2 \%)$ & \\
\hline Lacunar Stroke & $35(50 \%)$ & $4(5.8 \%)$ & \\
\hline Stroke of other determined cause & $0(0)$ & $0(0)$ & \\
\hline Stroke of undetermined etiology & $10(14.2 \%)$ & $14(20.6 \%)$ & \\
\hline $\begin{array}{r}\text { Difference of }>10 \% \text { between actual and } \\
\text { estimated body-weight }\end{array}$ & $6(8.6 \%)$ & $17(25 \%)$ & 0.010 \\
\hline
\end{tabular}

\section{Conclusion:}

Significant difference occurs between estimated and actual bodyweight in a considerable proportion of thrombolysed AIS patients. However, this discrepancy may not affect functional outcome or the risk of SICH 